\title{
Olig genes are upregulated in oligodendrocyte precursor cells in the injured central nervous system
}

\author{
Ken Iseki ${ }^{1,2}$, Seita Hagino ${ }^{3}$, Tetsuji Mori ${ }^{3}$, Yuxiang Zhang ${ }^{3}$, Nobuko Sakai ${ }^{3}$, \\ Sachihiko Yokoya ${ }^{3}$, Yasukazu Hozumi ${ }^{2}$, Kaoru Goto ${ }^{2}$, Akio Wanaka ${ }^{4}$, and \\ Choichiro Tase ${ }^{5}$
}

${ }^{I}$ Department of Emergency and Critical Care Medicine, ${ }^{2}$ Department of Anatomy and Cell Biology, Yamagata University, School of Medicine, Iida-Nishi 2-2-2, Yamagata 990-9585, Japan; ${ }^{3}$ Department of Cell Science, Institute of Biomedical Sciences, ${ }^{5}$ Department of Emergency Medicine, Fukushima Medical University, School of Medicine, 1 Hikarigaoka, Fukushima 960-1295, Japan; and ${ }^{4}$ Department of Anatomy, Nara Medical University, School of Medicine, Shijo-cho 840, Kashihara 634-8521, Japan

\begin{abstract}
Summary. The Olig gene family encodes basic helixloop-helix (bHLH) transcription factors and plays a critical role in oligodendrogenesis during development. We examined whether the Olig genes are involved in pathological conditions such as the injured brain or spinal cord by in situ hybridization. Olig1 mRNA was detected in cells surrounding necrotic tissue. The signal intensity of Olig1 mRNA gradually increased 2 to 7 days and decreased by 14 days after injury. Olig2 mRNA was expressed in a similar pattern and was colocalized with Olig1 mRNA. Similar results were obtained for the expression of Olig1 and Olig2 mRNAs in spinal cord injury. When we compared the mRNA expression pattern of Olig1 with that of NG2 proteoglycan and platelet-derived growth factor receptor $\alpha$ (PDGFR $\alpha)$, markers for oligodendrocyte precursor cells (OPCs), by double labeling in situ hybridization, Olig1 mRNA was co-localized with mRNAs for NG2 proteoglycan and PDGFRa 7 days after brain injury, suggesting that Olig mRNAs were expressed in OPCs. These findings suggest that the Olig transcription factors
\end{abstract}

Received May 20, 2011

Address for correspondence: Ken Iseki, M.D. Ph.D., Department of Emergency and Critical Care Medicine, Yamagata University, School of Medicine, Iida-Nishi 2-2-2, Yamagata City, Yamagata 990-9585, Japan

Tel: +81-23-628-5422, Fax: +81-23-628-5423

E-mail: ken@fmu.ac.jp regulate differentiation of oligodendrocytes in the injured CNS as well as in the developing CNS.

\section{Introduction}

Glial cells respond to central nervous system (CNS) injury and are transformed into a population of reactive cells, including reactive astrocytes, remyelinating oligodendrocytes and activated microglia (Rabchevsky et al., 2001). Oligodendrocyte precursor cells (OPCs), consisting of a $5-8 \%$ glial cell population in the intact adult CNS, are increased in number and recruited to a region surrounding necrotic tissue 3 to 5 days and decreased 7 to 10 days after injury (Levine et al., 2001). In the injured CNS, NG2 is upregulated in OPCs identified by markers such as NG2 proteoglycan, plateletderived growth factor receptor $\alpha(\mathrm{PDGFR} \alpha)$ and basic fibroblast growth factor receptor (bFGFR) (Stallcup et al., 1987; Levine et al., 2001).

As its name implies, OPCs are equivalent to the oligodendrocyte-type 2 astrocyte (O-2A) progenitor cells that can develop into either oligodendrocytes or type 2 astrocytes in vitro (Lee et al., 2000). In an in vivo developmental situation, however, O-2A progenitors preferentially differentiate into oligodendrocytes. A question then arises: Do O-2A progenitors or OPCs in the injured sites differentiate into oligodendrocytes or astrocytes or both? In certain demyelinating diseases such as multiple sclerosis (MS), incomplete remyelination is attributed to either a shortage or differentiation failure of 
OPCs (Wolswijk, 2002). Recent evidence suggests that OPCs in the MS lesion preferentially differentiate into astrocytes, but not into oligodendrocytes, due to an activated Notch signal (John et al., 2002). In this regard, differentiation mechanisms of OPCs in the adult injured brain are of particular interest.

The Olig gene family encodes basic helix-loop-helix (bHLH) transcription factors. Olig1 and Olig2, are expressed in the spinal cord and forebrain during development (Zhou et al., 2000), while Olig3 is not detected in the CNS (Takebayashi et al., 2000). Ectopic expression of Olig1 gene in progenitor cells promotes oligodendrocyte differentiation ( $\mathrm{Lu}$ et al., 2001). Genedisruption studies revealed that Olig1 and Olig2 govern the early differentiation process of oligodendrocytes (Lu et al., 2002; Zhou et al., 2002). Although the pivotal roles of Olig genes in oligodendrocyte development have been established, the expression and functions of Olig genes in the adult brain remain enigmatic. Previous studies have shown that Olig2 is upregulated in OPCs under pathological conditions in the adult brain (Fancy et al., 2004; Buffo et al., 2005; Talbott et al., 2005; Tatsumi et al., 2008) and that Olig2-positive progenitor cells differentiate into oligodendrocytes in demyelinated lesions (Islam, 2008). However, but it remains uncertain whether Olig1 is involved in pathological conditions. To investigate the mechanisms regulating such glial cell responses, we examined spatio-temporal expression patterns of Olig mRNAs in the injured CNS using markers for OPCs.

\section{Materials and Methods}

\section{Animal experiments and tissue preparation}

Adult male ICR mice were used in our experiments and all surgical procedures were approved according to the guidelines for animal experiments established by the institutional committee of Fukushima Medical University School of Medicine. Animal experiments were based on previous reports (Iseki et al., 2002; Hagino et al., 2003). Briefly, surgical procedures were performed under inhaled anesthesia of diethyl ether. Cryo-injury model was inflicted by placing a lead probe pre-chilled with liquid nitrogen on the cranium for $1 \mathrm{~min}$. Spinal cord injury model was made by the same technique as the brain injury after laminectomy at the eighth thoracic vertebra. Thirty mice were sacrificed under deep ether anesthesia at 2, 4, 7 and 14 days after surgery and perfused transcardially with phosphate buffered saline (PBS), followed by $4 \%$ paraformaldehyde (PFA). The brains or spinal cords were dissected, post-fixed in 4\% PFA for $24 \mathrm{~h}$ at $4^{\circ} \mathrm{C}$, and immersed in $30 \%$ sucrose in diethyl pyrocarbonate-treated PBS. They were embedded in an OCT compound (Tissue Tek, Torrance, CA, USA) and stored at $-80^{\circ} \mathrm{C}$ until use. Coronal sections $(10 \mu \mathrm{m}$ thick $)$ of the brains or sagittal sections of the spinal cords (10 $\mu \mathrm{m}$ thick) were cut on a cryostat and mounted onto silane-coated glass slides. Sections were then subjected to in situ hybridization.

\section{In situ hybridization}

In situ hybridization using a digoxigenin-labeled cRNA probe was performed as described previously (Nikaido et al., 2001; Iseki et al., 2002). Digoxigenin-labeled cRNA probes (antisense and sense probes) were made by in vitro transcription using Olig1, Olig2, NG2 and PDGFR $\alpha$. The NG2 and PDGFR $\alpha$ cDNAs were obtained from IMAGE consortium cDNA library. The Olig1 and Olig2 cDNAs were kindly provided by Dr. Zhou Q (Zhou et al., 2000). Genbank accession numbers for the cDNAs were as follows; AI019805 for NG2 and BE629400 for PDGFR $\alpha$. We confirmed the validity of the cDNAs by sequencing and no cross-reactivity between the probes by in situ hybridization on mouse embryo sections.

\section{Double-labeling fluorescence in situ hybridization}

Double-labeling in situ hybridization was performed as described previously (Iseki et al., 2002). Sections were hybridized with digoxigenin-labeled Olig2, NG2 or PDGFR $\alpha$ cRNA probes and FITC-labeled Olig1 cRNA probes. The Olig2, NG2 or PDGFR $\alpha$ mRNAs were visualized using a rhodamine-conjugated anti-digoxigenin antibody (1:100 dilution, Roche Products, Indianapolis, IN, USA). Olig1 mRNA was detected using an anti-FITC antibody conjugated to horseradish peroxidase (1:500, Roche Products) and was amplified using FITCconjugated tyramide (TSA Fluorescence System; NEN Life Science Products, Boston, MA, USA). Doublelabeled immunofluorescent sections were examined using an Olympus Fluoview FV300 confocal laser scanning microscope (Olympus, Tokyo, Japan).

\section{Results}

\section{Expression of Olig gene mRNAs in CNS injury}

Cryoinjury to the brain disrupted the blood brain barrier and induced edema followed by necrosis in our experimental model. Olig1 mRNA was increased in intensity primarily in the margin surrounding the necrotic region 7 days after injury (Fig. 1A, C). Similarly, Olig2 was also weakly expressed in the marginal region 7 days after injury 

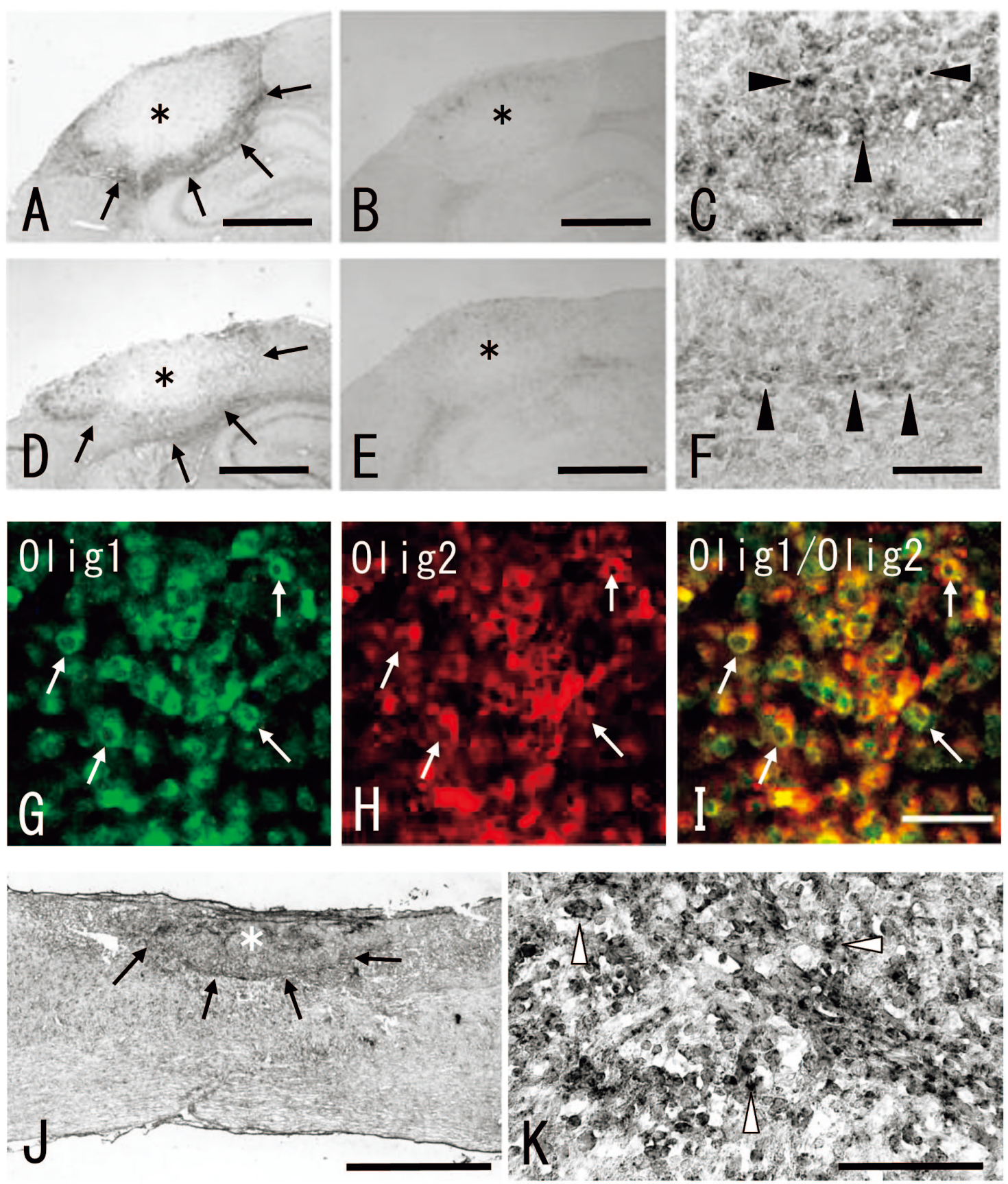

Fig. 1. In situ hybridization of Olig mRNAs at 7 days after CNS injury. (A-F) Messenger RNA transcripts for Olig1 (A) and Olig2 (D) are upregulated in the margin (arrows) of necrotic tissue (asterisks) of the injured brain. Higher magnification of the marginal zone (C for Olig1, F for Olig2) shows labeled cells (arrowheads). Incubation with sense probe for Olig1 gene (B) or Olig2 gene (E) generated no detectable signals. (G-I) Confocal images of double-labeling in situ hybridization show that Olig1 mRNA (G) is co-localized with Olig2 mRNA (H) in one and the same cells (arrows). (J, K) In spinal cord injury, Olig1 mRNA is similarly upregulated at 7 days after insult in the margin (arrows) of necrotic tissue (asterisk). Higher magnification shows labeled cells (K, arrowheads). Scale bars: $1 \mathrm{~mm}(\mathrm{~A}, \mathrm{~B}, \mathrm{D}, \mathrm{E}, \mathrm{J}), 100 \mu \mathrm{m}(\mathrm{C}, \mathrm{F}, \mathrm{K}), 50 \mu \mathrm{m}(\mathrm{I})$. 

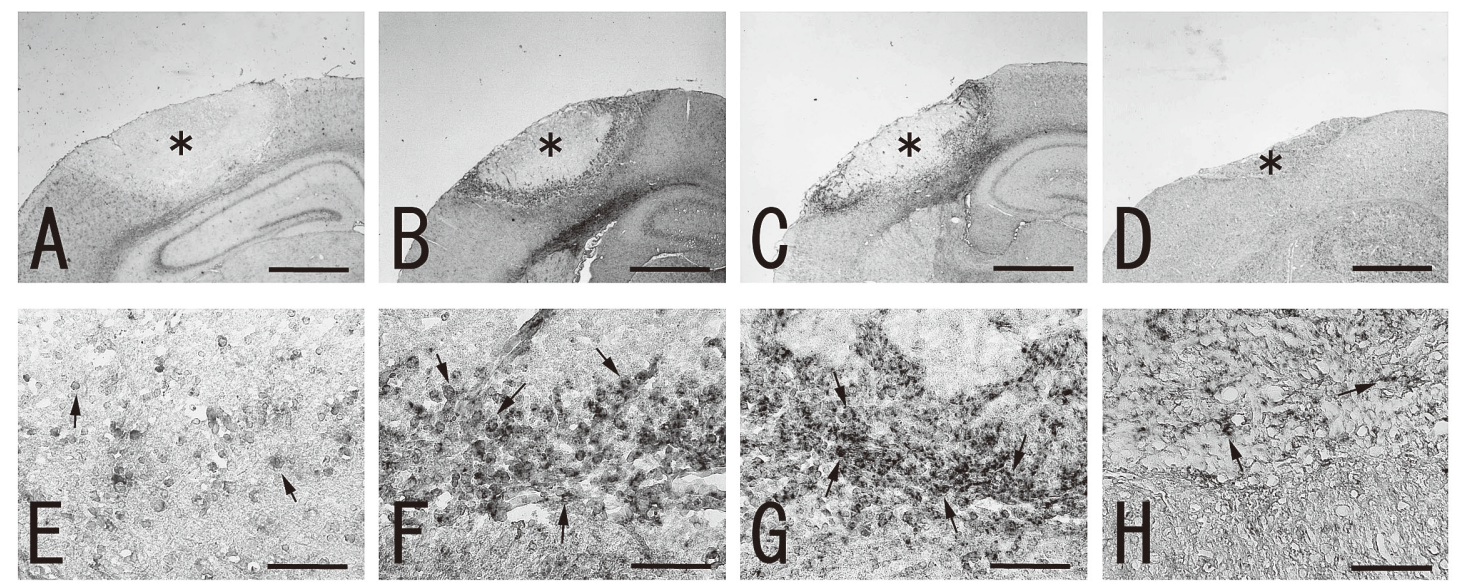

Fig. 2. Time-course of in situ hybridization of Olig1 mRNA expression after brain injury. Sections at 2, 4, 7 and 14 days after brain injury at low (A, B, C, D) and high (E, F, G, H) magnifications, respectively. The signal intensity for Olig1 mRNA gradually increases in the margin of the necrotic tissue (asterisks) from 2 to 7 days. It decreases by 14 days. High magnification shows labeled cells (arrowheads). Scale bars: $1 \mathrm{~mm}(\mathrm{~A}-\mathrm{D}), 100 \mu \mathrm{m}(\mathrm{E}-\mathrm{H})$.

(Fig. 1D, F). Control experiments with labeled sense strand probes gave no detectable signals in adjacent sections, confirming the specificity of the hybridization signals in this study (Fig. 1B, E). To examine whether Olig1 and Olig2 mRNAs are coexpressed or not, we performed double-labeling fluorescence in situ hybridization. Figures 1G-I showed that Olig1 and Olig2 mRNAs are co-localized in one and the same cell (Fig. 1G-I). In spinal cord injury, the expression pattern of Olig1 mRNA was observed in a similar fashion to that in brain injury (Fig. 1J, K).

We next examined the time course of Olig1 mRNA expression; we performed in situ hybridization at time points of 2, 4, 7, and 14 days after injury. Olig1 mRNA positive cells were detected on days 2-14 (Fig. 2). On day 2 to 4, Olig1 mRNA was weakly expressed in cells surrounding the necrotic tissue and reached a maximum level on day 7 (Fig. 2A-C). On day 14, necrotic tissue was largely absorbed where Olig1 mRNA expression was detected at low intensity (Fig. 2D).

\section{Olig1 mRNA is coexpressed with those of NG2 and PDGFR $\alpha$}

In the developing brain, NG2 and PDGFR $\alpha$ serve as wellknown markers for OPCs. To investigate whether Olig1 is expressed in OPCs, we compared the expression pattern of Olig1 mRNA with that of NG2 or PDGFR $\alpha$ by double labeling in situ hybridization. Olig1 mRNA was colocalized with NG2 proteoglycan core protein mRNA (Fig. 3A-C).
Furthermore, Olig1 mRNA was also coexpressed with PDGFR $\alpha$ mRNA in one and the same cell (Fig. 3D-F). These results suggest that Olig1 is expressed in OPCs in the injured brain.

\section{Discussion}

The present study reveals that Olig1 mRNA is coexpressed with Olig2 mRNA in OPCs that are labeled for either NG2 mRNA or PDGFR $\alpha$ mRNA in CNS injury. Previous studies have shown that NG2 and PDGFR $\alpha$ are reliable markers for OPCs in vivo (Levine et al., 2001) and that mRNAs for NG2 and PDGFR $\alpha$ are detected in not only the developing CNS but also in the white and gray matters of the adult CNS (Nishiyama et al., 1996), suggesting that OPCs remain in the adult CNS. In addition, it is shown that NG2-positive OPCs are increased in number and reach a peak at 7 days after CNS injury (Levine, 1994; Jones et al., 2002) and that PDGFRa mRNA expression is upregulated in a similar manner and peaks at 7 days after injury (Cook et al., 1998). In this regard, we reveal that the mRNA expression time course of Olig1 closely resembles that of NG2 and PDGFR $\alpha$. In the developing CNS, genes for Olig and PDGFR $\alpha$ are detected at the earliest phase of the oligodendrocyte lineage, which is followed by NG2 expression ( $\mathrm{Lu}$ et al., 2000; Zhou et al., 2000; Alberta et al., 2001). We detected upregulation of Olig1 mRNA as early as 2 days after injury, suggesting that in the injury paradigm Olig 

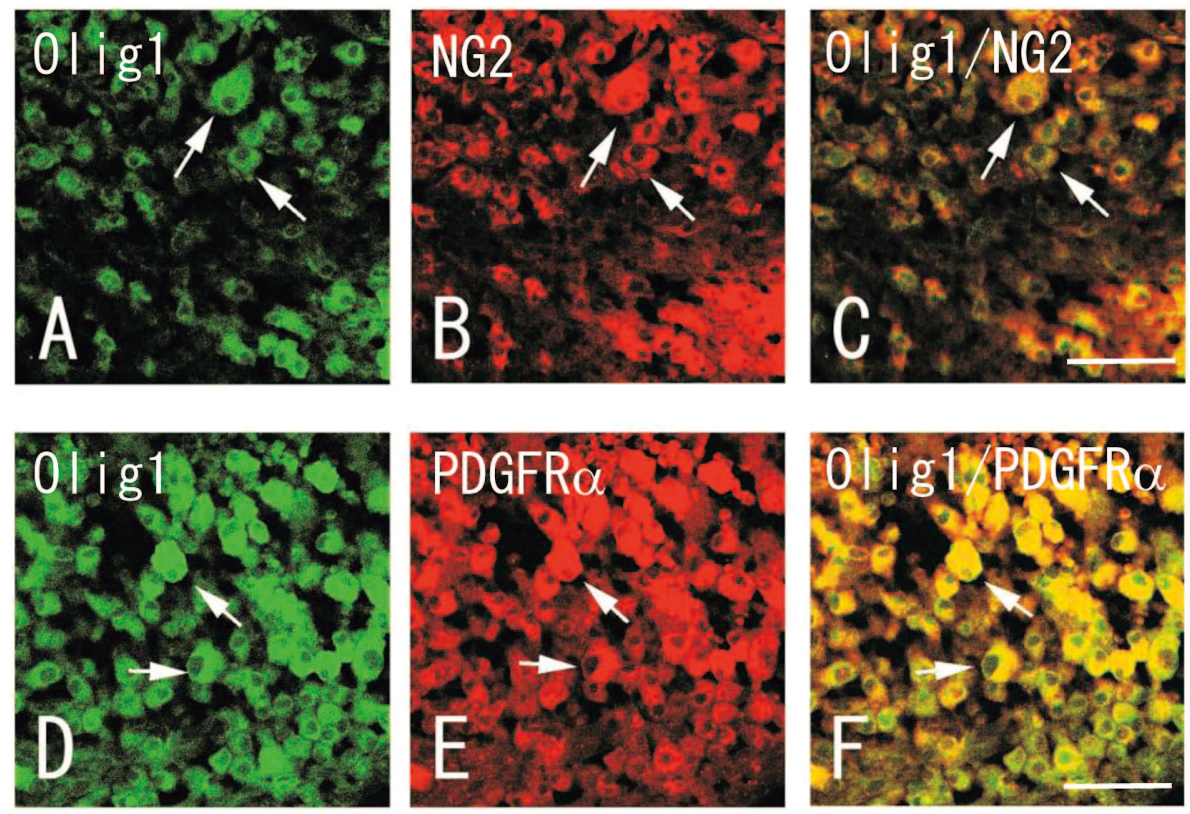

Fig. 3. Confocal images showing double-labeling in situ hybridization for Olig1 mRNA and oligodendrocyte precursor cell marker mRNAs at 7 days after brain injury. Olig1 mRNA is colocalized with both NG2 proteoglycan core protein mRNA (A-C) and PDGFR $\alpha$ mRNA (D-F) in one and the same cell (arrows). Scale bars: $50 \mu \mathrm{m}(\mathrm{C}, \mathrm{F})$.

genes may be an early-phase regulator of OPCs as in the developing CNS.

During development, Mash1, Hes5, and Id4 are shown to be involved in the differentiation of neural stem cells into OPCs. Among them Hes5 and Id4 inhibit oligodendrocyte differentiation (Kondo et al., 2000a; Kondo et al., 2000b; Raff et al., 2001). Although we examined the expression of these bHLH genes in our injury model, we found that their expressions are below the detection level (data not shown). Since $\operatorname{Id} 1, \mathrm{Id} 2$ and $\mathrm{Id} 3$ are reported to be expressed in spinal cord injury (Tzeng et al., 2001), these genes may substitute for Hes5 or Id4 in our injury model. These findings suggest that adult OPCs and/or the surrounding environment might be somewhat different between pathological conditions and developing brain. Another discrepancy between the adult injury paradigm and developmental situation is the expression of sonic hedgehog (shh), which, in embryonic CNS, is necessary and sufficient for the activation of Olig genes in OPC differentiation ( $\mathrm{Lu}$ et al., 2000; Alberta et al., 2001). However, we hardly observed the expression of shh and its receptors (Patched and Smoothend) in the injured brain (data not shown). The lack of shh expression may be ascribed to insufficient oligodendrocyte differentiation and poor remyelination in our injury model, which is frequently found in the adult CNS lesion (Alberta et al., 2001).

Recently, Olig-deficient mice have shed light on the functional roles of Olig genes (Alberta et al., 2001; Lu et al., 2002; Zhou et al., 2002). In Olig1 and Olig2 doubledeficient mice neither OPCs labeled by PDGFR $\alpha$ and Sox 10 nor mature oligodendrocytes labeled by myelin basic protein (MBP) and PLP/DM20 are detected in the brain and spinal cord (Zhou et al., 2002). In Olig2-null mice, on the other hand, OPCs labeled by PDGFR $\alpha$, Sox10, and Olig1 exist in the forebrain and hindbrain, although no OPCs are detected in the spinal cord. Furthermore, in Olig1-null mice both OPCs and mature oligodendrocytes are generated ( $\mathrm{Lu}$ et al., 2002). Taken these results together, it is suggested that Olig2 are required for OPC differentiation in the brain and spinal cord although Olig1 can compensate for the lack of Olig2 in oligodendrocytes in the brain but not in the spinal cord. Therefore, OPC differentiation may be controlled by Olig1 and Olig2 in a differential manner in different CNS regions. In this context, our present finding that shows coexpression of Olig1 and Olig2 mRNAs in OPC of the injured brain suggests that both Olig1 and Olig2 are 
required for the OPC proliferation and differentiation after injury. It should also be noted that Olig genes are good markers for OPCs in addressing the mechanisms of injury-response in the adult brain.

\section{Acknowledgements}

Authors thank Dr. Qiao Zhou for the Olig1 and Olig2 plasmids, and Ms. Mayumi Takeuchi and Ms. Hiromi Hashimoto for technical assistance. This work was supported by Grant-in Aid from the Ministry of Education, Science, Culture, Sports, Science and Technology (MEXT) of Japan (K.I.).

\section{References}

Alberta JA, Park SK, Mora J, Yuk D, Pawlitzky I, Iannarelli P, Vartanian T, Stiles CD, Rowitch DH: Sonic hedgehog is required during an early phase of oligodendrocyte development in mammalian brain. Mol Cell Neurosci 18: 434-441 (2001).

Buffo A, Vosko MR, Erturk D, Hamann GF, Jucker M, Rowitch D, Gotz M: Expression pattern of the transcription factor Olig2 in response to brain injuries: implications for neuronal repair. Proc Natl Acad Sci USA 102: 18183-18188 (2005).

Cook JL, Marcheselli V, Alam J, Deininger PL, Bazan NG: Temporal changes in gene expression following cryogenic rat brain injury. Brain Res Mol Brain Res 55: 9-19 (1998).

Fancy SPJ, Zhao C, Franklin RJM: Increased expression of Nkx2.2 and Olig2 identifies reactive oligodendrocyte progenitor cells responding to demyelination in the adult CNS. Mol Cell Neurosci 27: 247-254 (2004).

Hagino S, Iseki K, Mori T, Zhang Y, Hikake T, Yokoya S, Takeuchi M, Hasimoto H, Kikuchi S, Wanaka A: Slit and glypican-1 mRNAs are coexpressed in the reactive astrocytes of the injured adult brain. Glia 42: 130-138 (2003).

Iseki K, Hagino S, Mori T, Zhang Y, Yokoya S, Takaki H, Tase C, Murakawa M, Wanaka A: Increased syndecan expression by pleiotrophin and FGF receptor-expressing astrocytes in injured brain tissue. Glia 39: 1-9 (2002).

Islam MS, Tatsumi K, Okuda H, Shiosaka S, Wanaka A: Olig2-expressing progenitor cells preferentially differentiate into oligodendrocytes in cuprizone-induced demyelinated lesions. Neurochem Int 54: 192-198 (2009).

John GR, Shankar SL, Shafit-Zagardo B, Massimi A, Lee SC, Raine CS, Brosnan CF: Multiple sclerosis: reexpression of a developmental pathway that restricts oligodendrocyte maturation. Nat Med 8: 1115-1121 (2002).

Jones LL, Yamaguchi Y, Stallcup WB, Tuszynski MH: NG2 is a major chondroitin sulfate proteoglycan produced after spinal cord injury and is expressed by macrophages and oligodendrocyte progenitors. $J$ Neurosci 22: 2792-2803 (2002).

Kondo T, Raff M: Basic helix-loop-helix proteins and the timing of oligodendrocyte differentiation. Development 127: 2989-2998 (2000a).

Kondo T, Raff M: Oligodendrocyte precursor cells reprogrammed to become multipotential CNS stem cells. Science 289: 1754-1757 (2000b).

Lee JC, Mayer-Proschel M, Rao MS: Gliogenesis in the central nervous system. Glia 30: 105-121 (2000).

Levine JM: Increased expression of the NG2 chondroitinsulfate proteoglycan after brain injury. $J$ Neurosci 14 : 4716-4730 (1994).

Levine JM, Reynolds R, Fawcett JW: The oligodendrocyte precursor cell in health and disease. Trends Neurosci 24: 39-47 (2001).

Lu QR, Cai L, Rowitch D, Cepko CL, Stiles CD: Ectopic expression of Olig1 promotes oligodendrocyte formation and reduces neuronal survival in developing mouse cortex. Nat Neurosci 4: 973-974 (2001).

Lu QR, Sun T, Zhu Z, Ma N, Garcia M, Stiles CD, Rowitch DH: Common developmental requirement for Olig function indicates a motor neuron/oligodendrocyte connection. Cell 109: 75-86 (2002).

Lu QR, Yuk D, Alberta JA, Zhu Z, Pawlitzky I, Chan J, McMahon AP, Stiles CD, Rowitch DH: Sonic hedgehog - regulated oligodendrocyte lineage genes encoding bHLH proteins in the mammalian central nervous system. Neuron 25: 317-329 (2000).

Nikaido T, Yokoya S, Mori T, Hagino S, Iseki K, Zhang Y, Takeuchi M, Takaki H, Kikuchi S, Wanaka A: Expression of the novel transcription factor OASIS, which belongs to the CREB/ATF family, in mouse embryo with special reference to bone development. Histochem Cell Biol 116: 141-148 (2001).

Nishiyama A, Lin XH, Giese N, Heldin CH, Stallcup WB: Co-localization of NG2 proteoglycan and PDGF alphareceptor on $\mathrm{O} 2 \mathrm{~A}$ progenitor cells in the developing rat brain. J Neurosci Res 43: 299-314 (1996).

Rabchevsky AG, Smith GM: Therapeutic interventions following mammalian spinal cord injury. Arch Neurol 58: 721-726 (2001).

Raff M, Apperly J, Kondo T, Tokumoto Y, Tang D: Timing cell-cycle exit and differentiation in oligodendrocyte development. Novartis Found Symp 237: 100-107 (2001).

Stallcup WB, Beasley L: Bipotential glial precursor cells of the optic nerve express the NG2 proteoglycan. $J$ 
Neurosci 7: 2737-2744 (1987).

Takebayashi H, Yoshida S, Sugimori M, Kosako H, Kominami R, Nakafuku M, Nabeshima Y: Dynamic expression of basic helix-loop-helix Olig family members: implication of Olig2 in neuron and oligodendrocyte differentiation and identification of a new member, Olig3. Mech Dev 99: 143-148 (2000).

Talbott JF, Loy DN, Liu Y, Qiu MS, Bunge MB, Rao MS, Whittemore SR: Endogenous Nkx2.2 $/$ Olig2 ${ }^{+}$ oligodendrocyte precursor cells fail to remyelinate the demyelinated adult rat spinal cord in the absence of astrocytes. Exp Neurol 192: 11-24 (2005).

Tatsumi K, Takebayashi H, Manabe T, Tanaka KF, Makinodan M, Yamauchi T, Makinodan E, Matsuyoshi H, Okuda H, Ikenaka K, Wanaka A: Genetic fate mapping of Olig2 progenitors in the injured adult cerebral cortex reveals preferential differentiation into astrocytes. J Neurosci Res 86: 3494-502 (2008).

Tzeng SF, Bresnahan JC, Beattie MS, de Vellis J: Upregulation of the HLH Id gene family in neural progenitors and glial cells of the rat spinal cord following contusion injury. J Neurosci Res 66: 11611172 (2001).

Wolswijk G: Oligodendrocyte precursor cells in the demyelinated multiple sclerosis spinal cord. Brain 125: 338-349 (2002).

Zhou Q, Anderson DJ: The bHLH transcription factors OLIG2 and OLIG1 couple neuronal and glial subtype specification. Cell 109: 61-73 (2002).

Zhou Q, Wang S, Anderson DJ: Identification of a novel family of oligodendrocyte lineage-specific basic helixloop-helix transcription factors. Neuron 25: 331-343 (2000). 